\title{
A Thin, Angle-insensitive, Broadband Microwave Absorber Based on Metamaterial
}

\author{
KeTao Zhou ${ }^{1, a}$, Jin Pan ${ }^{2, b}$ and Rong Pan ${ }^{2, c}$ \\ ${ }^{1}$ College of computer Science and Electronic Engineering, \\ HuNan University, ChangSha 410082, China; \\ ${ }^{2}$ College of computer Science and Electronic Engineering, \\ HuNan University, ChangSha 410082, China; \\ ${ }^{2}$ College of computer Science and Electronic Engineering, \\ HuNan University, ChangSha 410082, China. \\ aktzhou@hnu.edu.cn, bpanjin@hnu.edu.cn, cpanrong@hnu.edu.cn
}

Keywords: Broadband absorber, thin absorber, angle-insensitive absorber, single-square loops, field-localization.

\begin{abstract}
In this paper, we have presented a simple model for making thin, angle-insensitive, broadband metamaterial absorber in microwave frequency. The proposed structure consists of a square loop imprinted on a aluminum-backed dielectric substrate. Numerical results show that a -10dB absorption bandwidth from 4.77 to $9.52 \mathrm{GHz}$ has been realized under normal incidence. The distributions of electromagnetic field have been illustrated at the low-frequency peak of $5.4 \mathrm{GHz}$ and high-frequency peak of $8.0 \mathrm{GHz}$ to analyze the absorption mechanism of the proposed structure. And we find that the absorption dependent on the thickness of substrate and the length of square loop hugely. Moreover, the magnitude distributions of electric field along the direction of y-axis on proposed absorber has been presented with incident angles ranging from $0^{0}$ to $60^{\circ}$. Because rotational symmetric properties of the structure, the performance of the absorber is insensitive to the angle of the incident waves, which indicated the prospect of future application in the field of microwave absorber based on metamaterials.
\end{abstract}

\section{Introduction}

In recent years, metamaterials(MMs) [1-5] have triggered a notable scientific investigation with an expanding impact on modern optical device due to its unnatural electromagnetic properties, MMs has many applications like perfect filter [6], lens [7], cloaking [8], antenna system [9]etc. It is well known that most of the MMs are periodically arranged with metallic structure that is much smaller than the operating wavelength in size. The electromagnetic responses of MMs can be characterized as homogeneous media, and the effective optical parameters such as permittivity and permeability can be calculated by tailing the configuration of MMs. Recently, absorbers based on metamaterial have been developed ranging from microwave frequency to THz [10], MIR [11], and the visible realm [12] due to its near perfect absorption, ultrathin thickness, wide bandwidth properties, easier manufacturing and increasing application [13-16].

However, most of the metamaterial absorbers(MAs) reported in the literature are generally composed of a metallic patch and ground metal plane parted by dielectric substrate. Meanwhile, MAs suffer from narrow operating bandwidth as they designed with characteristic of ultrathin and near perfect absorption [13], although some broadband MMs [2-5,12] have already been presented, it is only limited to absorb microwave with higher frequency or the pattern is too complicated to be produced, and some absorber designs also comprise multiple vertically stacked metallic layers, but the thickness is also a major limitation in those designs, Thus it is a challenge to design a MMs with lower frequency absorption and simpler construction. 
In this paper, a square loop structure in a single unit cell have been proposed which operates around the middle of the microwave C-band(4.77-9.52GHz), exhibits remarkable ultrathin properties, broadband absorption and independence on the angle of incident radiation. Meanwhile, by regulating the thickness of dielectric layer and the length of square loop can tuning the absorption of this structure. The merits of the optimized absorber are thoroughly validated via different length and thick setups, The distributions of electromagnetic field at the absorption peaks have been illustrated to analyze the absorption mechanism of the broadband absorber. In addition, the designed structure has been studied for different angles of incident radiation, while some possible future extensions are also discussed finally.

\section{Design of the Structure.}
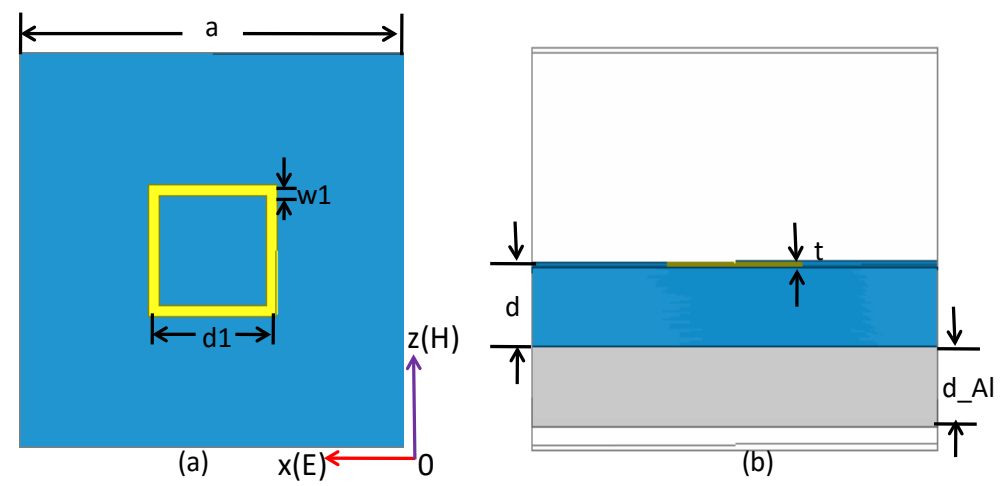

Fig. 1. single-square loop absorber (a) front view (b) side view

(unit mm: d1=4.0, w1=0.33, $\mathrm{a}=12, \mathrm{t}=0.01, \mathrm{~d}=2, \mathrm{~d} \_\mathrm{Al}=2$ )

The front view of the proposed structure is shown in Fig. 1(a), where the directions of electric, magnetic field are also shown, the structure of square loop made with copper printed on a dielectric substrate. The substrate has a thickness of $2 \mathrm{~mm}$ and a conductivity of 0 . the thickness of copper films are $0.01 \mathrm{~mm}$ with conductivity $\sigma_{1}=5.8 \times 10^{7} \mathrm{~S} / \mathrm{m}$ [5] and other dimensions are as shown in Fig. 1(a). The ground plane is consisted of aluminum (Fig.1(b)) with conductivity $\sigma_{2}=3.8 \times 10^{7} \mathrm{~S} / \mathrm{m}$, whereas the opposite face of the dielectric spacer is covered with a aluminum layer in order to ensure zero transmission when the incident electromagnetic through the structure. Additionally, periodic boundary conditions are applied along the $\mathrm{x}$ - and $\mathrm{z}$-axis in order to model an infinite array of unit cells.

\section{Numerical Calculations and Discussion}

For our analysis, all simulations are carried out through High Frequency Structure Simulator (HFSS) [14]by considering a single unit cell. A three-dimensional simulation model is shown in Fig. 2(a). Two-dimensional periodic boundary condition was imposed on the unit cell, and the incident wave was modeled as a Wave Port above the unit cell along y-axis.
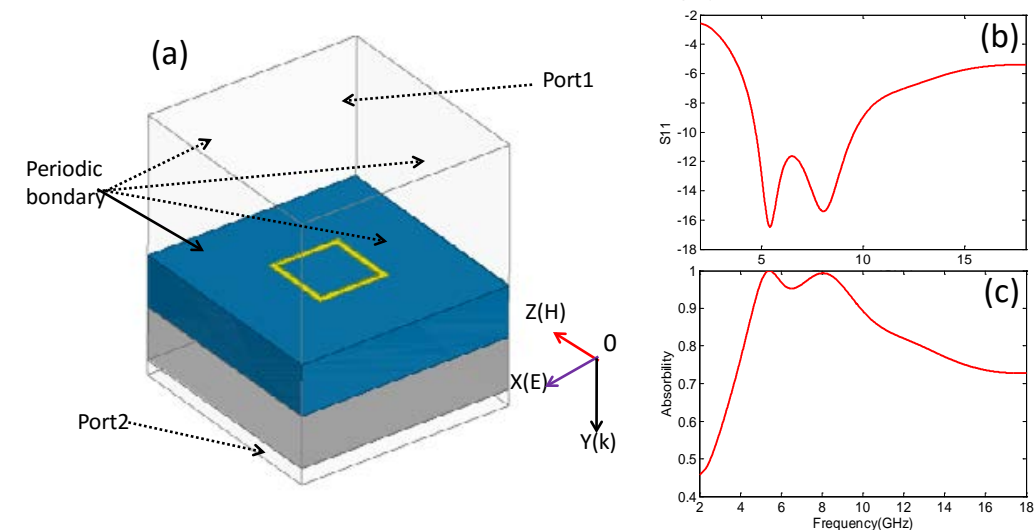

Fig. 2. (a) Unit-cell model in HFSS. (b)Reflection coefficient. (c)Absorbility 
Starting with arbitrary dimensions and considering a normally incident plane wave propagating along the $\mathrm{y}$-axis direction with its component of electric-field polarized along $\mathrm{x}$-axis and magnetic-field polarized along z-axis, a genetic algorithm is employed to reach the optimal unit cell parameters for maximum absorption near the middle of the C-band. The absorption of the structure is calculated from the values of the simulated scattering parameters through its definition as $A(\omega)=1-R(\omega)-T(\omega)$, with $R(\omega)=\left|S_{11}\right|^{2}$ and $T(\omega)=\left|S_{21}\right|^{2}$ being the reflectance and transmittance, respectively. Since $T(\omega)=0$ due to the aluminum plane, the absorption at frequency of $\omega$ can be maximized.

As illustrated in Fig.2(b), the reflection coefficient of unit cell under normal incidence with electric field polarized along x-axis. We clearly see that the proposed absorber has wide bandwidth ranging from 4.77 to $9.52 \mathrm{GHz}$, and the bottom of reflection coefficient distributed at both sides of $6.5 \mathrm{GHz}$. At the frequency of $5.4 \mathrm{GHz}$, the reflection coefficient is as low as $-17 \mathrm{~dB}$, while at $8.0 \mathrm{GHz}$, the reflection coefficient is $-15.8 \mathrm{~dB}$, it means that at the frequency of 5.4 and $8.0 \mathrm{GHz}$ there will appears absorption peak, as Fig.2(c) shown above.

From the simulations of our designed absorber, the absorption are greater than $99 \%$ at point of 5.4 and $8.0 \mathrm{GHz}$, the bandwidth of absorption above $90 \%$ almost reach $4.75 \mathrm{GHz}$. and then, it also reveals that the electric and magnetic are strongly in resonance around 5.4 and $8.0 \mathrm{GHz}$, due to near-perfect impedance-matched to the free space, which lead to near-zero reflection. In order to understand the physical mechanism of the absorption mechanism better, the distributions of electromagnetic field in the sides of the proposed MAs has been plotted at the two absorption peaks point of 5.4 and $8.0 \mathrm{GHz}$ (Fig. 3).

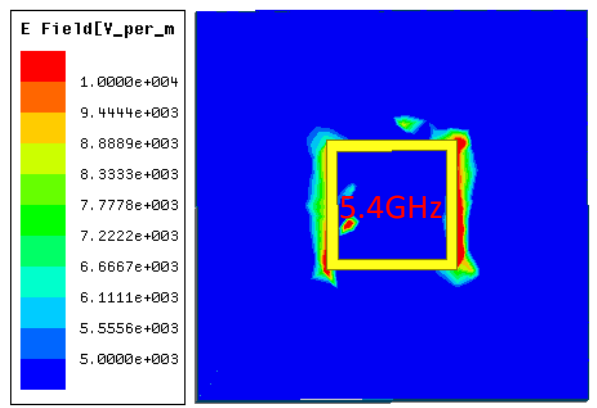

(a)

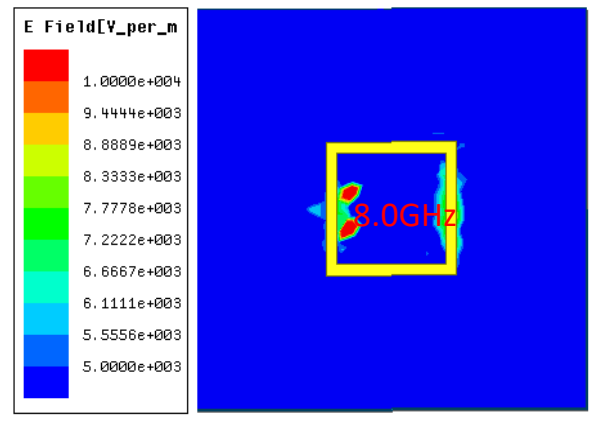

(c)

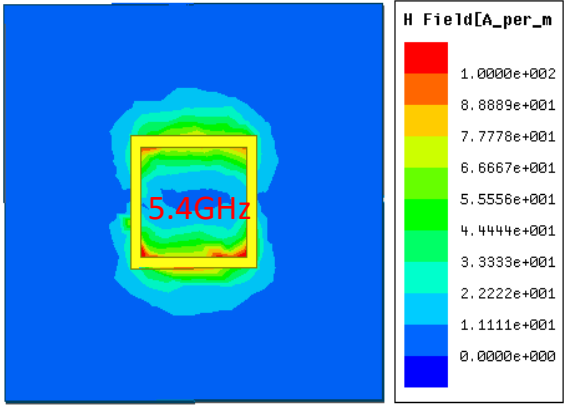

(b)

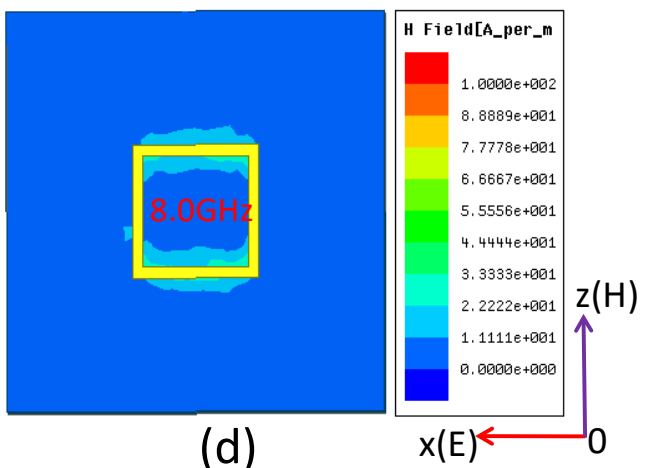

(d)

Fig.3 Simulated electric field distribution at (a) 5.4 and (c) $8.0 \mathrm{GHz}$ and magnetic field distribution at (b) 5.4 and (d) $8.0 \mathrm{GHz}$ of the proposed absorber structure.

By analyzing the field distribution, it is not hard to observe that at the lower resonance of $5.4 \mathrm{GHz}$, The electric field in the absorber is mainly focused on the two edge parallel to z-axis of the square loop. On the contrary, the magnetic field is primary localized on the two edge perpendicular to z-axis. At the electromagnetic resonance of higher frequency, the distribution of electromagnetic field is mostly the same as the one at lower frequency.

Due to the thickness of dielectric substrate will influence the couple of square-loop and ground metal plane, regulating the thickness of substrate can achieve different absorption effects, as shown in Fig.4. 


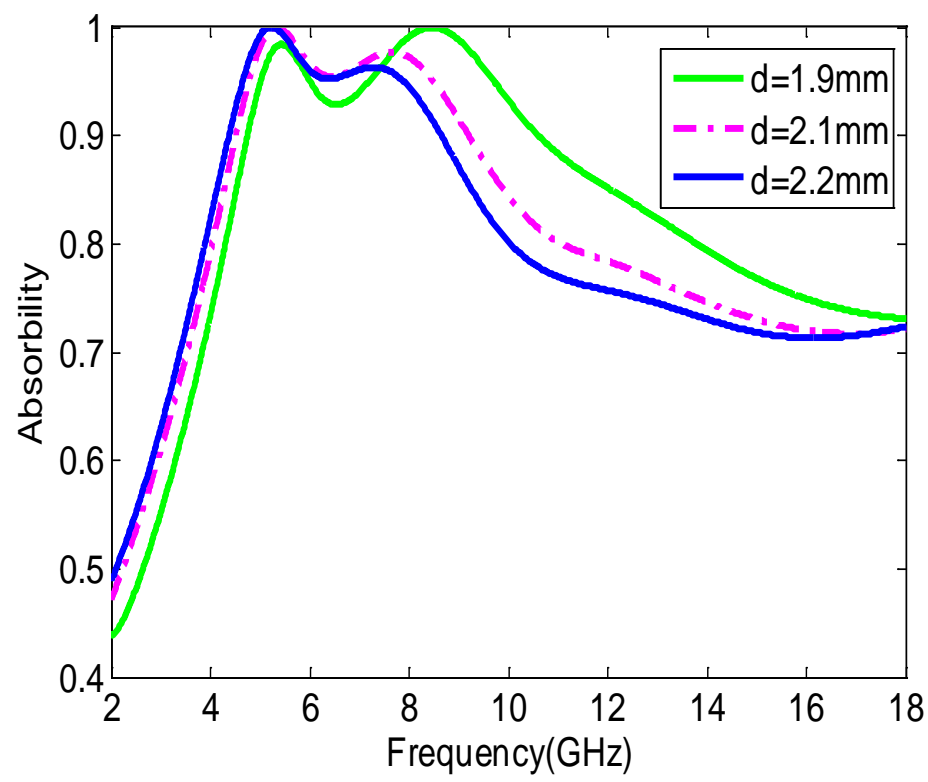

Fig.4 Absorbility for different d

From Fig.4, we can see that as d increasing, the whole curve of absorption moves toward the direction of lower frequency, especially in the vicinity of absorption peak. At the same time, the absorption peak at lower frequency become bigger with $\mathrm{d}$ increasing, while the peak of absorption at higher frequency is not monotonic as d changes. In addition, with d get longer , the bandwidth of absorption above $90 \%$ is decreased. Therefore, the absorption peak and the bandwidth of absorption can be adjusted by tuning the thickness of dielectric substrate.

Another critical parameter discussed here is the length of the square-loop influence on absorption. In this simulation, we presented different length with $\mathrm{d} 1=4.2 \mathrm{~mm}, \mathrm{~d} 1=4.4 \mathrm{~mm}$ and $\mathrm{d} 1=4.6 \mathrm{~mm}$ respect to absorption, as shown in Fig.5.

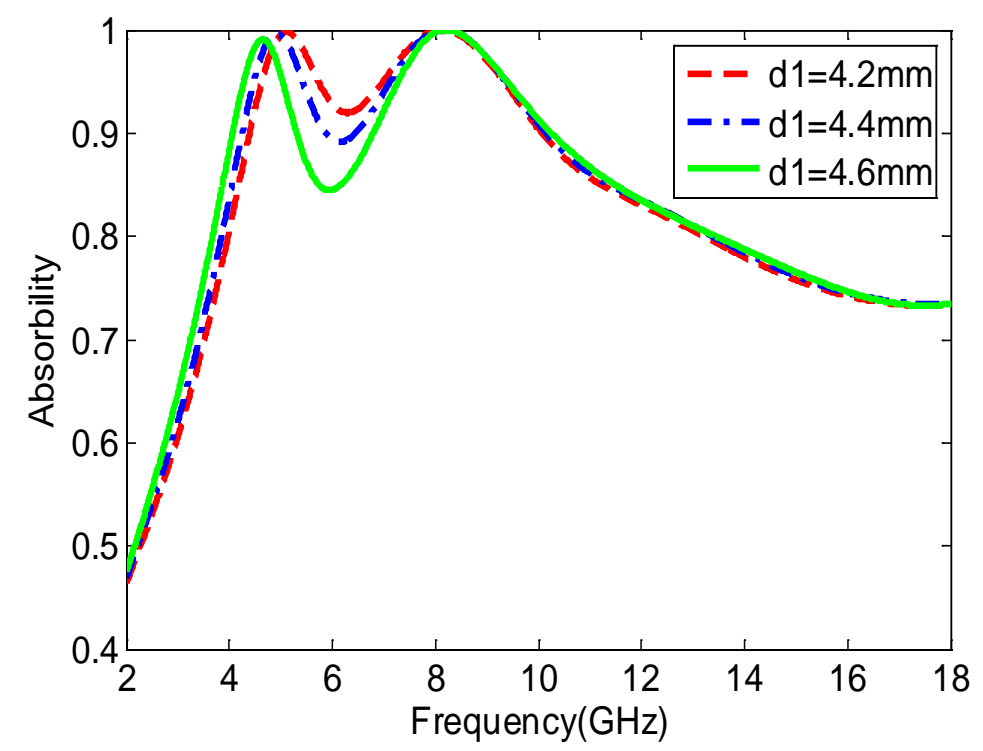

Fig.5 Absorbility for different d1

From the analysis of Fig.5, it is easy to get the conclusion that when the length of square-loop changes, the absorption peak at higher frequency remains almost consistent, well the absorption peak at lower frequency starts damping and move to the direction of lower frequency.

In the section above, we have just simulated the absorption under the condition of normal incidence. In practical application, electromagnetic waves are usually incident onto absorbers with any angle, so it is necessary to obtain the distribution of electromagnetic field with oblique incidence. The distribution for TE with incident angle ranging from $0^{\circ}$ (Fig.3(a)) to $60^{\circ}$ with $15^{\circ}$ changes interval are shown in Fig.6. 


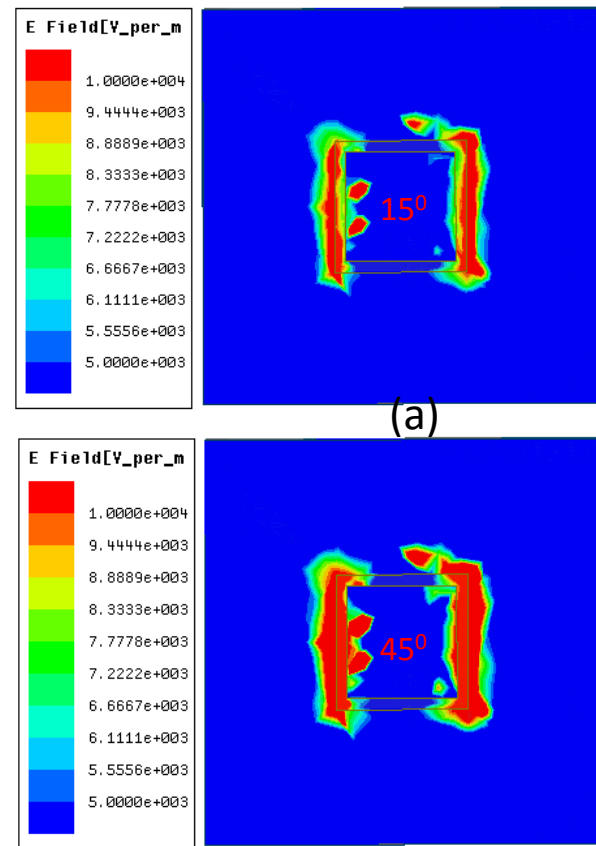

(c)

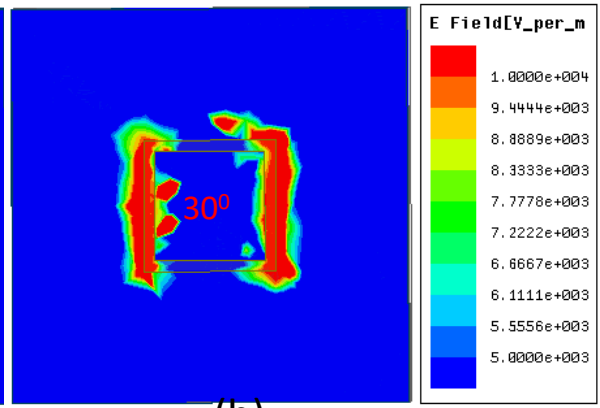

(b)

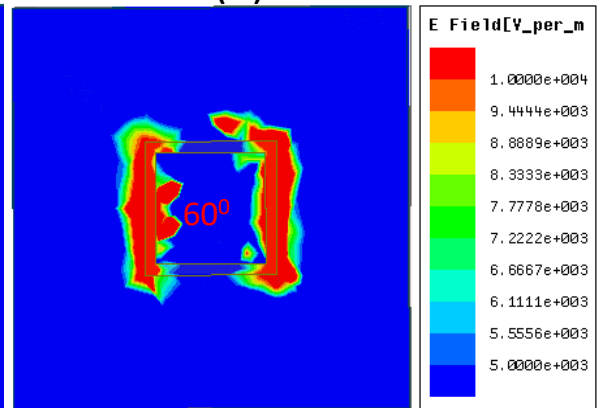

(d)

Fig.6 Electric field-location for different incidence angles

Surprisingly, our findings indicated that the field-localization almost maintain invariant as the angle of incident wave changing from $0^{\circ}$ to $60^{\circ}$, which means the absorber is complete with a fully functional of angle insensitive. As a result, no matter how the angle of incident wave changes, the electric field is mainly focused on the parallel side of the square loop.

\section{Summary}

In this paper, a thin, angle-insensitive, broadband metamaterial absorber in the microwave regime has been presented with a square loop. The simulation shows that the absorption above $90 \%$ for frequency range from 4.77 to $9.52 \mathrm{GHz}$, which means the absorber had an excellent broadband property. Tuning parameters of the optimized model, the bandwidth of absorption as well as absorption peak can be regulated. With the help of numerical simulation, the electromagnetic field distribution at the resonance frequency was studied, which strengthen us to understand the field distribution of square loop at the point of absorption peak. Therefore, the proposed model is a good alternative for field-localization and broadband microwave absorption, we believe that the proposed structure may have a bright future in the field of controlling microwave absorption and radar elimination.

\section{References}

[1] Landy, N. I., et al. "Perfect metamaterial absorber." Physical review letters 100 (2008): 207402.

[2] Tseng, Chao-Hsiung, and Chih-Lin Chang. "A broadband quadrature power splitter using metamaterial transmission line." Microwave and Wireless Components Letters, IEEE 18 (2008): 25-27.

[3] Ghosh, Saptarshi, et al. "An Ultrawideband Ultrathin Metamaterial Absorber Based on Circular Split Rings." Antennas and Wireless Propagation Letters, IEEE 14 (2015): 1172-1175.

[4] Dimitriadis, Alexandros, Nikolaos Kantartzis, and Theodoros Tsiboukis. "A polarization-/angle-insensitive, bandwidth-optimized, metamaterial absorber in the microwave regime." Applied Physics A 109 (2012): 1065-1070. 
[5] Liu, Xiao-Xin, et al. "A broadband polarization-insensitive metamaterial absorber based on multi-mode resonance." Antennas and Propagation (APCAP), 2014 3rd Asia-Pacific Conference on. IEEE, 2014.

[6] Gil, Marta, et al. "Composite right/left-handed metamaterial transmission lines based on complementary split-rings resonators and their applications to very wideband and compact filter design." Microwave Theory and Techniques, IEEE Transactions on 55 (2007): 1296-1304.

[7] Kundtz, Nathan, and David R. Smith. "Extreme-angle broadband metamaterial lens." Nature materials 9 (2010): 129-132.

[8] Alu, Andrea, and Nader Engheta. "Plasmonic and metamaterial cloaking: physical mechanisms and potentials." Journal of Optics A: Pure and Applied Optics 10 (2008): 093002.

[9] Metz, Carsten. "Phased array metamaterial antenna system." U.S. Patent No. 6,958,729. (2005).

[10] Chen, Hou-Tong, et al. "Active terahertz metamaterial devices." Nature 444 (2006): 597-600.

[11] Liu, Xianliang, et al. "Taming the blackbody with infrared metamaterials as selective thermal emitters." Physical review letters 107 (2011): 045901.

[12] Cheng, Yongzhi, et al. "Adjustable low frequency and broadband metamaterial absorber based on magnetic rubber plate and cross resonator."Journal of Applied Physics 115 (2014): 064902.

[13] Bhattacharyya, Somak, Saptarshi Ghosh, and Kumar Vaibhav Srivastava. "An ultra-thin polarization independent metamaterial absorber for triple band applications." Applied Electromagnetics Conference (AEMC), 2013 IEEE. IEEE, 2013.

[14] Li, Mei, et al. "An ultrathin and broadband radar absorber using resistive FSS." Antennas and Wireless Propagation Letters, IEEE 11 (2012): 748-751.

[15] Cheng, Yongzhi, et al. "Perfect metamaterial absorber based on a split-ring-cross resonator." Applied Physics A 102 (2011): 99-103.

[16] Ding, Fei, et al. "Ultra-broadband microwave metamaterial absorber." Applied Physics Letters 100 (2012): 103506. 